\title{
Effects of early aldosterone antagonism on cardiac remodeling in rats with aortic stenosis-induced pressure overload
}

\author{
M.P. Okoshi ${ }^{\text {a,* }}$, M.D.M. Cezar ${ }^{\text {b }}$, R.M. Iyomasa ${ }^{\text {a }}$, M.B. Silva ${ }^{\text {a }}$, L.C.O. Costa ${ }^{\text {a }}$, P.F. Martinez ${ }^{\text {c }}$, D.H.S. Campos ${ }^{\text {a }}$, \\ R.L. Damatto a,b ${ }^{\text {a, M.F. Minicucci }}{ }^{\text {a }}$, A.C. Cicogna ${ }^{\text {a }}$, K. Okoshi ${ }^{\text {a }}$ \\ a Department of Internal Medicine, Botucatu Medical School, Sao Paulo State University, UNESP, Brazil \\ ${ }^{\mathrm{b}}$ Itapeva Social and Agrarian Sciences College, FAIT, Itapeva, SP, Brazil \\ c Federal University of Mato Grosso do Sul, Campo Grande, Brazil
}

\section{A R T I C L E I N F O}

\section{Article history:}

Received 20 April 2016

Received in revised form 29 July 2016

Accepted 30 July 2016

Available online 1 August 2016

\section{Keywords:}

Spironolactone

Myocardial fibrosis

Cardiac hypertrophy

Ventricular function

Papillary muscle

\begin{abstract}
A B S T R A C T
Aldosterone plays a pivotal role in the pathophysiology of systolic heart failure. However, whether early aldosterone antagonism improves cardiac remodeling during persistent pressure overload is unsettled. We evaluated the effects of aldosterone antagonist spironolactone on cardiac remodeling in rats with ascending aortic stenosis (AS).

Methods: Three days after inducing AS, weaning rats were randomized to receive spironolactone (AS-SPR, $20 \mathrm{mg} / \mathrm{kg} / \mathrm{day}$ ) or no drug (AS) for 18 weeks, and compared with sham-operated rats. Myocardial function was studied in isolated left ventricular (LV) papillary muscles. Statistical analyses: ANOVA or Kruskal-Wallis tests.

Results: Echocardiogram showed that LV diastolic (Sham 8.73 \pm 0.57 ; AS $8.30 \pm 1.10$; AS-SPR 9.19 $\pm 1.15 \mathrm{~mm}$ ) and systolic (Sham $4.57 \pm 0.67$; AS $3.61 \pm 1.49$; AS-SPR $4.62 \pm 1.48 \mathrm{~mm}$ ) diameters, left atrial diameter (Sham $5.80 \pm 0.44 ;$ AS $7.15 \pm 1.22$; AS-SPR $8.02 \pm 1.17 \mathrm{~mm}$ ), and LV mass were higher in AS-SPR than AS. Posterior wall shortening velocity (Sham $38.5 \pm 3.8$; AS $35.6 \pm 5.6$; AS-SPR $31.1 \pm 3.8 \mathrm{~mm} / \mathrm{s}$ ) was lower in AS-SPR than Sham and AS; E/A ratio was higher in AS-SPR than Sham. Developed tension was lower in AS and AS-SPR than Sham. Time to peak tension was higher in AS-SPR than Sham and AS after post-rest contraction. Right ventricle weight was higher in AS-SPR than AS, suggesting more severe heart failure in AS-SPR than AS. Interstitial collagen fractional area and myocardial hydroxyproline concentration were higher in AS than Sham. Metalloproteinase- 2 and -9 activity, evaluated by zymography, did not differ between groups.

Conclusion: Early spironolactone administration causes further hypertrophy in cardiac chambers, and left ventricular dilation and dysfunction in rats with AS-induced chronic pressure overload.
\end{abstract}

(c) 2016 Elsevier Ireland Ltd. All rights reserved.

\section{Introduction}

Aldosterone is a mineralocorticoid hormone involved in renal sodium and potassium homeostasis and blood pressure modulation. Increased systemic and myocardial concentrations of aldosterone are associated with deleterious cardiac effects. Experimental studies have shown that aldosterone directly stimulates myocyte growth and strongly induces myocardial fibrosis [1,2]. The harmful effects of aldosterone on the cardiovascular system also include myocyte apoptosis, myocardial oxidative stress and electrical remodeling, vascular injury, endothelial dysfunction, ventricular arrhythmia, and sudden death [3, 4]. After the pioneer studies by Pitt et al. [5-7], aldosterone antagonists started to be evaluated in different cardiac aggression models, which

\footnotetext{
* Corresponding author at: Departamento de Clinica Medica, Faculdade de Medicina de Botucatu, UNESP Rubiao Junior, S/N 18618-970, Botucatu, SP, Brazil.

E-mail address: mpoliti@fmb.unesp.br (M.P. Okoshi).
}

showed that they prevent or attenuate left ventricular (LV) structural, functional, and molecular changes [8-11]. In clinical settings, aldosterone blockers reduced mortality and hospitalizations in patients with systolic heart failure of any cause [5,7], and in patients with systolic dysfunction after myocardial infarction [6]. Therefore, aldosterone blockade is now recommended for symptomatic systolic heart failure patients [12]. In contrast, recent studies have shown that aldosterone blockade failed to reduce cardiovascular morbimortality [13] or improve maximal exercise capacity, symptoms, or quality of life in heart failure patients with preserved ejection fraction [14]. The mechanisms responsible for the lack of response to aldosterone blockers in this situation are not clear.

Chronic pressure overload is a major cause of heart failure with preserved or reduced ejection fraction. During sustained pressure overload, cardiac remodeling is mainly characterized by myocyte hypertrophy and interstitial fibrosis $[15,16]$. Stable cardiac hypertrophy is usually maintained for a long period and may progress to a decompensated 
state with left ventricular dilation and systolic dysfunction [17,18]. The role of aldosterone antagonism on the development of cardiac hypertrophy during persistent pressure overload is not clear. In spontaneously hypertensive rats with LV hypertrophy and no heart failure features, aldosterone antagonist spironolactone reduced the frequency of heart failure development, improved myocardial function, and attenuated myocardial fibrosis independent of blood pressure levels [8]. In this study we tested the hypothesis that early aldosterone blockade improves cardiac remodeling during chronic pressure overload. Therefore, we evaluated the effects of spironolactone on cardiac structures, ventricular and myocardial function, and myocardial fibrosis in rats when administration was initiated three days after ascending aortic stenosis induction. In this model, 3-4 week-old rats are subjected to a clip placement around the ascending aorta [19]. After clip placement, aorta diameter is preserved; as rats grow, stenosis progressively develops [20,21]. Therefore, spironolactone was started before LV hypertrophy was established.

\section{Materials and methods}

\subsection{Experimental groups}

Male Wistar rats weighing 80-100 g were purchased from the Central Animal House at Botucatu Medical School, UNESP. All animals were housed in a temperature controlled room at $23^{\circ} \mathrm{C}$ and kept on a 12-hour light/dark cycle. Food and water were supplied ad libitum. All experiments and procedures were approved by the Animal Experimentation Ethics Committee of Botucatu Medical School, UNESP, SP, Brazil.

The animals underwent median thoracotomy under anesthesia with intraperitoneal ketamine hydrochloride $(50 \mathrm{mg} / \mathrm{kg}$ ) and xylidine hydrochloride $(10 \mathrm{mg} / \mathrm{kg})$. Aortic constriction was induced by placing a $0.6 \mathrm{~mm}$ stainless-steel clip on the ascending aorta via a thoracic incision according to a previously described method [22]. Age-matched sham operated rats were used as controls. Three days after surgery, the rats were randomly assigned into three groups: Sham $(n=22)$, aortic stenosis $(A S, n=41)$ and aortic stenosis treated with spironolactone (AS-SPR, $\mathrm{n}=36$ ). Treatment was initiated three days after surgery and kept up for 18 weeks. Spironolactone was added to rat chow at a dosage of $20 \mathrm{mg} / \mathrm{kg} / \mathrm{day}$. At the end of the experimental period, rats were subjected to transthoracic echocardiogram and euthanized the next day.

\subsection{Echocardiographic study}

Cardiac structures and left ventricular (LV) function were evaluated by transthoracic echocardiogram using a commercially available echocardiograph (General Electric Medical Systems, Vivid S6, Tirat Carmel, Israel) equipped with a 5-11.5 MHz multifrequency transducer as previously described [23-25]. Rats were anesthetized by intramuscular injection with a mixture of ketamine $(50 \mathrm{mg} / \mathrm{kg})$ and xylazine $(0.5 \mathrm{mg} / \mathrm{kg})$. A twodimensional parasternal short-axis view of the left ventricle (LV) was obtained at the level of the papillary muscles. M-mode tracings were obtained from short-axis views of the LV at or just below the tip of the mitral-valve leaflets, and at the level of the aortic valve and left atrium. M-mode images of the LV were printed on a black-and-white thermal printer (Sony UP-890MD) at a sweep speed of $100 \mathrm{~mm} / \mathrm{s}$. All LV structures were manually measured by the same observer (KO) according to the leading-edge method of the American Society of Echocardiography [26]. Values obtained were the mean of at least five cardiac cycles on M-mode tracings. The following structural variables were measured: LV diastolic and systolic dimensions (LVDD and LVSD, respectively), LV diastolic and systolic posterior wall thickness (LVDPWT and LVSPWT, respectively), LV diastolic and systolic septal wall thickness (LVDSWT and LVSSWT, respectively), aortic diameter (AO), and left atrium (LA) diameter. Left ventricular mass (LVM) was calculated using the formula $\left[(\text { LVDD }+ \text { PWT }+ \text { SWT })^{3}-(\text { LVDD })^{3}\right] \times 1.04$. Relative wall thickness $($ RWT $)$ was calculated as $2 \times$ PWT / LVDD. LV function was assessed by the following parameters: endocardial fractional shortening (EFS), midwall fractional shortening (MWFS), posterior wall shortening velocity (PWSV), early and late diastolic mitral inflow velocities (E and A waves), E/A ratio, E-wave deceleration time (EDT), and isovolumetric relaxation time (IVRT).

\subsection{Myocardial functional study}

Two days after echocardiographic study, myocardial contractile performance was evaluated in isolated LV papillary muscle preparations as previously described $[27,28]$. Rats were anesthetized with pentobarbital sodium, $50 \mathrm{mg} / \mathrm{kg}$, intraperitoneally, and decapitated. Hearts were quickly removed and placed in oxygenated Krebs-Henseleit solution at $28{ }^{\circ} \mathrm{C}$. LV anterior or posterior papillary muscle was dissected free, mounted between two spring clips, and placed vertically in a chamber containing Krebs-Henseleit solution at $28{ }^{\circ} \mathrm{C}$ and oxygenated with a mixture of $95 \% \mathrm{O}_{2}$ and $5 \% \mathrm{CO}_{2}(\mathrm{pH} 7.38)$. The composition of the Krebs-Henseleit solution in mM was as follows: $118.5 \mathrm{NaCl}, 4.69 \mathrm{KCl}, 1.25$ $\mathrm{CaCl}_{2}, 1.16 \mathrm{MgSO}_{4}, 1.18 \mathrm{KH}_{2} \mathrm{PO}_{4}, 5.50$ glucose, and $25.88 \mathrm{NaHCO}_{3}$. The spring clips were attached to a Kyowa model 120T-20B force transducer and a lever system, which allowed for muscle length adjustment. Preparations were stimulated 12 times/min at a voltage $10 \%$ above threshold.

After a 60 -min period, during which the preparations were permitted to shorten while carrying light loads, muscles were loaded to contract isometrically and stretched to the apices of their length-total tension curves $\left(\mathrm{L}_{\max }\right)$. After a 5 -min period, during which preparations performed isotonic contractions, muscles were again placed under isometric conditions, and the apex of the length-total tension curve was determined. A 15 minute period of stable isometric contraction was imposed prior to the experimental period. One isometric contraction was then recorded for later analysis.

The following parameters were measured from isometric contraction: peak of developed tension (DT, g/mm ${ }^{2}$ ), resting tension $\left(\mathrm{RT}, \mathrm{g} / \mathrm{mm}^{2}\right.$ ), maximum rate of tension development $\left(+\mathrm{dT} / \mathrm{dt}, \mathrm{g} / \mathrm{mm}^{2} / \mathrm{s}\right)$, maximum rate of tension decline $\left(-\mathrm{dT} / \mathrm{dt}, \mathrm{g} / \mathrm{mm}^{2} / \mathrm{s}\right)$, and time to peak of tension (TPT). To evaluate contractile reserve, papillary muscle mechanical performance was evaluated at basal conditions and after the following inotropic stimulation: post-rest contraction, extracellular $\mathrm{Ca}^{2+}$ concentration increase, and $\beta$-adrenergic agonist isoproterenol addition to the nutrient solution [29].

Papillary muscle cross-sectional area (CSA) was calculated from muscle weight and length by assuming cylindrical uniformity and a specific gravity of 1.0. All force data were normalized for the muscle CSA. After dissecting papillary muscle, atria and ventricles were separated and weighed. Atria and left and right ventricular weights were normalized to body weight.

\subsection{Morphologic study}

Transverse LV sections were fixed in $10 \%$ buffered formalin and embedded in paraffin. Five-micrometer-thick sections were stained with hematoxylin-eosin and collagenspecific stain picrosirius red (Sirius red F3BA in aqueous saturated picric acid) [30]. In each heart, at least 50 myocyte diameters were measured as the shortest distance between borders drawn across the nucleus. On average, 20 microscopic fields were used to quantify interstitial collagen fractional area. Perivascular collagen was excluded from this analysis. Measurements were performed using a Leica microscope (magnification $40 \times$ ) attached to a video camera and connected to a computer equipped with image analysis software (Image-Pro Plus 3.0, Media Cybernetics, Silver Spring, MD, USA).

\subsection{Myocardial hydroxyproline concentration}

Myocardial hydroxyproline (HOP) concentration was assessed to estimate tissue collagen content. HOP was measured in LV tissue as previously described [31,32]. Briefly, the tissue was dried using a Speedvac Concentrator SC 100 attached to a refrigerated condensation trap (TRL 100) and vacuum pump (VP 100, Savant Instruments, Inc., Farmingdale, NY, USA). Dry tissue weight was measured and samples were hydrolyzed overnight at $100{ }^{\circ} \mathrm{C}$ with $6 \mathrm{~N} \mathrm{HCl}(1 \mathrm{~mL} / 10 \mathrm{mg}$ dry tissue). A $50 \mu \mathrm{L}$ aliquot of hydrolysate was transferred to an Eppendorf tube and dried in the Speedvac Concentrator. One milliliter of deionized water was added and the sample transferred to a tube with a Teflon screw cap. One milliliter of potassium borate buffer ( $\mathrm{pH}$ 8.7) was added to maintain constant $\mathrm{pH}$ and the sample was oxidized with $0.3 \mathrm{~mL}$ of chloramine $\mathrm{T}$ solution at room temperature for $20 \mathrm{~min}$. The addition of $1 \mathrm{~mL}$ of 3.6 M sodium thiosulfate and thorough mixing for $10 \mathrm{~s}$ stopped the oxidative process. The solution was then saturated with $1.5 \mathrm{~g} \mathrm{KCl}$. The tubes were heated in boiling water for $20 \mathrm{~min}$. After cooling to room temperature, the aqueous layer was extracted with $2.5 \mathrm{~mL}$ of toluene. One and a half milliliters of toluene extract were transferred to a $12 \times 75 \mathrm{~mm}$ test tube. Then $0.6 \mathrm{~mL}$ of Ehrlich's reagent was added and the color allowed to develop for $30 \mathrm{~min}$. Absorbances were read at $565 \mathrm{~nm}$ against a reagent blank. Deionized water and $20 \mu \mathrm{g} / \mathrm{mL}$ HOP were used as the blank and standard, respectively.

\subsection{Metalloproteinase activity}

Matrix metalloproteinase (MMP)-2 and -9 activity was determined as previously reported [33]. In brief, analysis samples were prepared by dilution in extraction sample buffer with $50 \mathrm{mM}$ Tris, pH 7.4; $0.2 \mathrm{M} \mathrm{NaCl}$; $0.1 \%$ Triton $\mathrm{X}$, and $10 \mathrm{mM} \mathrm{CaCl}_{2}$. In sample protein was quantified by the Bradford method. Samples with $20 \mu \mathrm{g}$ of protein were then diluted in application buffer with $0.5 \mathrm{M}$ Tris, pH $6.8 ; 100 \%$ glycerol, and $0.05 \%$ bromophenol blue, and loaded into wells of $8 \%$ SDS-polyacrylamide containing $1 \%$ gelatin. Electrophoresis was run in a Bio-Rad apparatus at $80 \mathrm{~V}$ for $2 \mathrm{~h}$. Gel was removed, washed twice with $2.5 \%$ Triton-X-100, and washed with $50 \mathrm{mM}$ Tris, $\mathrm{pH}$ 8.4. Gel was then incubated at $37^{\circ} \mathrm{C}$ overnight in activation solution with $50 \mathrm{mM}$ Tris, pH $8.4 ; 5 \mathrm{mM} \mathrm{CaCl}_{2}$, and $\mathrm{ZnCl}_{2}$. Staining was performed for $2 \mathrm{~h}$ with $0.5 \%$ coomassie blue and destaining in $30 \%$ methanol and $10 \%$ acetic acid at room temperature until clear bands over a dark background were observed. The gels were photographed and the intensity of gelatinolytic action (clear bands) was analyzed in UVP, UV, White Darkhon image analyzer.

\subsection{Statistical analysis}

Data are expressed as mean \pm standard deviation or median and 25th and 75th percentiles. Comparisons between groups were performed by one way analysis of variance (ANOVA) followed by Tukey test or Kruskal-Wallis followed by Dunn test. Mortality was assessed by log-rank test (Kaplan Meier). Statistical significance was accepted at the level of $\mathrm{p}<0.05$. 


\section{Results}

During the experiment, AS and AS-SPR groups had mortality rates of $51.2 \%$ and $41.7 \%$, respectively $(\mathrm{p}=0.28$ ). No rat from Sham group died during the experimental period. Body weight (BW) and anatomic parameters are shown in Table 1. Final body weight was lower in AS and AS-SPR than Sham. LV and atria weight, in absolute or normalized to body weight values, and right ventricle weight were higher in AS and AS-SPR than Sham and higher in AS-SPR than AS. In rats, the increase in atria weight and particularly the right ventricle weight are strong predictors of heart failure [34,35]. Therefore, the higher atria and right ventricle weight in AS-SPR suggests that spironolactone induced a more severe degree of heart failure.

Aldosterone antagonist doses vary between different experimental studies [36-38]. In previous works, we have observed that $20 \mathrm{mg} / \mathrm{kg} / \mathrm{day}$ of spironolactone does not reduce arterial blood pressure in normotensive (data not shown) or hypertensive rats [8,39]. In addition, lower doses such as $10 \mathrm{mg}$ daily of spironolactone had beneficial cardiovascular effects [37]. As the purpose of this study was to use a dose associated with cardiovascular effects but with no change in blood pressure, the rats received $20 \mathrm{mg} / \mathrm{kg} /$ day of spironolactone. After subjecting young rats to ascending stenosis, heart failure and ensuing death usually start to occur $18-22$ weeks after surgery $[20,22,40]$. Therefore, to avoid excessively high mortality rates, we established 18 weeks as the period to treat our rats.

Table 2 shows structural cardiac parameters and Fig. 1 shows illustrative LV M-mode echocardiograms. LV diastolic diameter-to-body weight ratio, LV walls thickness, left atrial diameter, LV mass, and LV relative thickness were higher in AS and AS-SPR than Sham. LV diastolic (Sham $8.73 \pm 0.57$; AS $8.30 \pm 1.10$; AS-SPR $9.19 \pm 1.15 \mathrm{~mm}$ ) and systolic (Sham 4.57 \pm 0.67 ; AS $3.61 \pm 1.49$; AS-SPR $4.62 \pm 1.48 \mathrm{~mm}$ ) diameters, left atrial diameter (Sham $5.80 \pm 0.44$; AS $7.15 \pm 1.22$; AS-SPR $8.02 \pm 1.17 \mathrm{~mm}$ ), and LV mass were higher in AS-SPR than AS. Table 3 shows LV functional parameters. Endocardial fractional shortening was higher in AS than Sham. E wave was higher and E-wave deceleration time and isovolumetric relaxation time lower in AS and AS-SPR than Sham. Posterior wall shortening velocity (Sham $38.5 \pm 3.8$; AS $35.6 \pm 5.6$; AS-SPR $31.1 \pm 3.8 \mathrm{~mm} / \mathrm{s}$ ) was lower in AS-SPR than Sham and AS; E/A ratio was higher in AS-SPR than Sham.

Table 4 shows basal papillary muscle data. Developed tension and $+\mathrm{dT} / \mathrm{dt}$ were lower and resting tension higher in both AS and AS-SPR than Sham. Time to peak tension was higher in AS-SPR than Sham. Contractile reserve was evaluated after positive inotropic stimulation (Table 5). The $+\mathrm{dT} / \mathrm{dt}$ was lower in AS and AS-SPR than Sham after all inotropic stimulation. Developed tension did not differ between groups after post-rest contraction and extracellular calcium increase to $2.5 \mathrm{mM}$, and was lower in AS and AS-SPR than Sham after isoproterenol addition to nutrient solution. Time to peak tension was higher in AS-SPR than Sham after all inotropic stimulation and was higher in AS-SPR than AS

Table 1

Anatomic data.

\begin{tabular}{llll}
\hline & $\begin{array}{l}\text { Sham } \\
(\mathrm{n}=22)\end{array}$ & $\begin{array}{l}\text { AS } \\
(\mathrm{n}=20)\end{array}$ & $\begin{array}{l}\text { AS-SPR } \\
(\mathrm{n}=21)\end{array}$ \\
\hline Initial BW $(\mathrm{g})$ & $82.8 \pm 7.6$ & $79.8 \pm 2.0$ & $79.4 \pm 2.3$ \\
Final BW $(\mathrm{g})$ & $479 \pm 56$ & $415 \pm 40^{*}$ & $413 \pm 36^{*}$ \\
LVW $(\mathrm{g})$ & $0.86 \pm 0.11$ & $1.16 \pm 0.16^{*}$ & $1.35 \pm 0.19^{*}, \#$ \\
LVW/BW $(\mathrm{g} / \mathrm{kg})$ & $1.80 \pm 0.11$ & $2.79 \pm 0.25^{*}$ & $3.27 \pm 0.37^{*}, \#$ \\
RVW $(\mathrm{g})$ & $0.26 \pm 0.04$ & $0.31 \pm 0.13$ & $0.40 \pm 0.12^{*}, \#$ \\
RVW/BW $(\mathrm{g} / \mathrm{kg})$ & $0.55 \pm 0.05$ & $0.75 \pm 0.32^{*}$ & $0.96 \pm 0.29^{*}, \#$ \\
Atria $(\mathrm{g})$ & $0.11 \pm 0.02$ & $0.18 \pm 0.01^{*}$ & $0.27 \pm 0.01^{*}, \#$ \\
Atria/BW $(\mathrm{g} / \mathrm{kg})$ & $0.22 \pm 0.02$ & $0.43 \pm 0.18^{*}$ & $0.64 \pm 0.23^{*}, \#$ \\
\hline
\end{tabular}

Data are mean \pm standard deviation. Sham: control sham-operated rats; AS: aortic stenosis; AS-SPR: aortic stenosis treated with spironolactone; BW: body weight; LVW: left ventricle weight; RVW: right ventricle weight. ANOVA and Tukey.

* $\mathrm{p}<0.05$ vs Sham.

\# $\mathrm{p}<0.05$ vs AS.
Table 2

Echocardiographic structural cardiac data.

\begin{tabular}{llll}
\hline & $\begin{array}{l}\text { Sham } \\
(\mathrm{n}=22)\end{array}$ & $\begin{array}{l}\text { AS } \\
(\mathrm{n}=20)\end{array}$ & $\begin{array}{l}\text { AS-SPR } \\
(\mathrm{n}=21)\end{array}$ \\
\hline LVDD $(\mathrm{mm})$ & $8.73 \pm 0.57$ & $8.30 \pm 1.10$ & $9.19 \pm 1.15^{\#}$ \\
LVDD/BW $(\mathrm{mm} / \mathrm{kg})$ & $18.1 \pm 1.79$ & $20.1 \pm 2.92^{*}$ & $22.2 \pm 3.10^{*}, \#$ \\
LVSD $(\mathrm{mm})$ & $4.57 \pm 0.67$ & $3.61 \pm 1.49^{*}$ & $4.62 \pm 1.48^{\#}$ \\
LVDPWT $(\mathrm{mm})$ & $1.56 \pm 0.07$ & $2.14 \pm 0.28^{*}$ & $2.25 \pm 0.27^{*}$ \\
LVSPWT $(\mathrm{mm})$ & $2.80 \pm 0.25$ & $3.81 \pm 0.35^{*}$ & $3.70 \pm 0.32^{*}$ \\
LVDSWT $(\mathrm{mm})$ & $1.59 \pm 0.07$ & $2.13 \pm 0.28^{*}$ & $2.25 \pm 0.26^{*}$ \\
LVSSWT $(\mathrm{mm})$ & $2.59 \pm 0.19$ & $3.11 \pm 0.34^{*}$ & $3.22 \pm 0.40^{*}$ \\
AO $(\mathrm{mm})$ & $3.90 \pm 0.28$ & $4.06 \pm 0.31$ & $3.99 \pm 0.18$ \\
LA $(\mathrm{mm})$ & $5.80 \pm 0.44$ & $7.15 \pm 1.22^{*}$ & $8.02 \pm 1.17^{*}, \#$ \\
LA/AO & $1.49 \pm 0.11$ & $1.77 \pm 0.33^{*}$ & $2.01 \pm 0.27^{*}, \#$ \\
LA/BW $(\mathrm{mm} / \mathrm{kg})$ & $12.0 \pm 1.15$ & $17.3 \pm 3.59^{*}$ & $19.4 \pm 2.73^{*}, \#$ \\
LVM $(\mathrm{g})$ & $1.05 \pm 0.13$ & $1.52 \pm 0.54^{*}$ & $1.90 \pm 0.53^{*}, \#$ \\
LVMI $(\mathrm{g} / \mathrm{kg})$ & $2.17 \pm 0.26$ & $3.65 \pm 1.24^{*}$ & $4.58 \pm 1.35^{*}, \#$ \\
RWT & $0.18 \pm 0.01$ & $0.26 \pm 0.03^{*}$ & $0.25 \pm 0.04^{*}$ \\
\hline
\end{tabular}

Data are mean \pm standard deviation. Sham: control sham-operated rats; AS: aortic stenosis; AS-SPR: aortic stenosis treated with spironolactone; LVDD and LVSD: left ventricular (LV) diastolic and systolic diameters, respectively; BW: body weight; LVDPWT and LVSPWT: LV diastolic and systolic posterior wall thickness, respectively; LVDSWT and LDSSWT: LV diastolic and systolic septal wall thickness, respectively; AO: aorta diameter; LA: left atrial diameter; LVM: LV mass; LVMI: LV mass index; RWT: relative wall thickness. ANOVA and Tukey.

* $\mathrm{p}<0.05$ vs Sham

\# $\mathrm{p}<0.05$ vs AS.

after post-rest contraction [Sham 165 (160-170); AS 168 (165-180); AS-SPR 188 (175-195) ms]. LV morphometric and biochemical data are shown in Table 6. Myocyte diameters were higher in AS and ASSPR than Sham and did not differ between AS and AS-SPR. Interstitial collagen fractional area (Sham $2.29 \pm 0.77$; AS $5.13 \pm 3.37$; AS-SPR $3.14 \pm 3.25 \%$; Fig. 2) and myocardial hydroxyproline concentration were higher in AS than Sham; in AS-SPR, these parameters had values between those of the Sham and AS groups and did not differ significantly from either group. Metalloproteinase- 2 and -9 activity did not differ between groups.

\section{Discussion}

In this study, we observed that early administration of aldosterone antagonist spironolactone unexpectedly increased cardiac hypertrophy and dilation, and impaired LV function in rats with aortic stenosisinduced chronic LV pressure-overload.

Ascending aortic stenosis in rats has been used to promote the gradual development of LV pressure overload and hypertrophy, similarly to human chronic pressure overload. In this model, a clip is placed around the ascending aorta in 3-4 week-old rats. Immediately after clip placement aorta diameter is preserved; as rats grow, stenosis and LV hypertrophy progressively develops [20-22]. Rats remain compensated for approximately 18 to 28 weeks [40]. In this study, spironolactone was initiated three days after surgery; therefore, the aldosterone blockade was started before the development of LV hypertrophy.

The addition of spironolactone to rat chow did not change food intake as AS and AS-SPR groups had similar body weights at the end of the experiment. Body weight was approximately $13.5 \%$ lower in AS and AS-SPR than Sham, showing the occurrence of cardiac cachexia and the severity of cardiac injury. Cardiac cachexia has been considered an independent predictor of mortality [41] and has been well characterized in rats with aortic stenosis-induced heart failure [42]. Probably due to the small sample size to evaluate mortality, this parameter did not significantly differ between AS and AS-SPR groups.

The echocardiographic study showed that both aortic stenosis groups presented left atrial dilation and LV concentric hypertrophy. The increased $\mathrm{E}$ and decreased E-wave deceleration time and isovolumetric relaxation time in AS and AS-SPR are in accordance with the anatomic parameters indicating higher filling pressures [43]. Hypertrophy in ascending aortic stenosis is usually better adaptive than in 

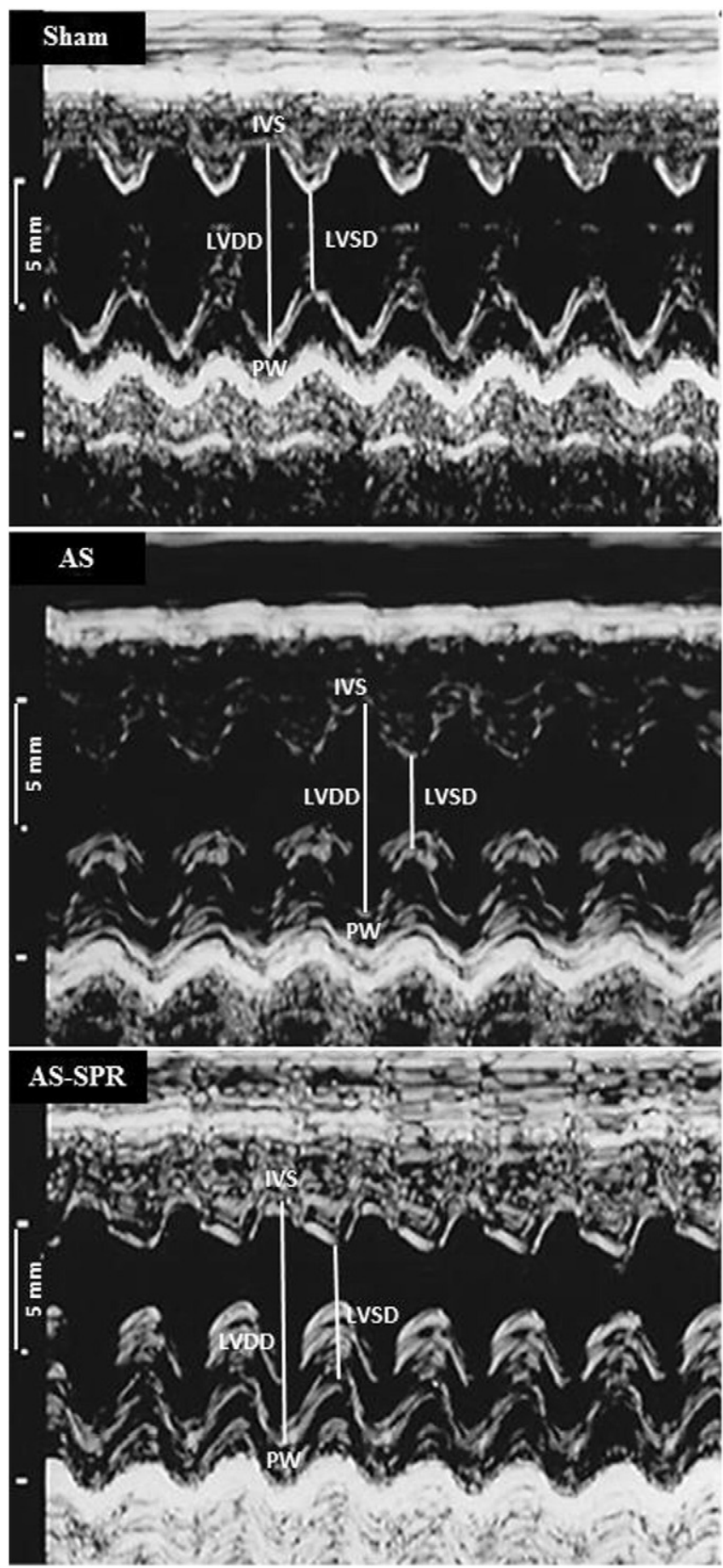

Fig. 1. Illustrative left ventricle M-mode echocardiograms. LVDD and LVSD: left ventricular (LV) diastolic and systolic diameters, respectively; PW: LV posterior wall; IVS: interventricular septum; Sham: control sham-operated rats; AS: aortic stenosis; AS-SPR: aortic stenosis treated with spironolactone.

descending aortic narrowing, as sustained loading occurring early in systole is followed by less concentric hypertrophy [44]. The fact that heart rate differed between AS and Sham groups may suggest that the level of anesthesia was not the same for all rats. However, we believe that the influence of anesthesia on cardiac function was reduced by the great number of animals included in each group.

Unexpectedly, we observed that spironolactone increased left atrial and LV dilation, and LV hypertrophy over and above values in the AS group. Despite AS-SPR having larger LV systolic and diastolic diameters than AS, LV hypertrophy remained concentric. Functionally, AS-SPR had
Table 3

Echocardiographic left ventricular functional parameters.

\begin{tabular}{llll}
\hline & $\begin{array}{l}\text { Sham } \\
(\mathrm{n}=22)\end{array}$ & $\begin{array}{l}\text { AS } \\
(\mathrm{n}=20)\end{array}$ & $\begin{array}{l}\text { AS-SPR } \\
(\mathrm{n}=21)\end{array}$ \\
\hline HR (beats/min) & $285 \pm 30$ & $322 \pm 56^{*}$ & $308 \pm 26$ \\
MWFS (\%) & $29.5 \pm 3.4$ & $32.9 \pm 7.2$ & $29.7 \pm 5.8$ \\
EFS $(\%)$ & $47.8 \pm 5.6$ & $57.8 \pm 12.5^{*}$ & $50.7 \pm 10.6$ \\
PWSV (mm/s) & $38.5 \pm 3.8$ & $35.6 \pm 5.6$ & $31.1 \pm 3.8^{*}, \#$ \\
E-wave (cm/s) & $78.1 \pm 7.8$ & $103.7 \pm 31.6^{*}$ & $118.3 \pm 28.9^{*}$ \\
A-wave (cm/s) & $47.7 \pm 10.0$ & $55.9 \pm 27.6$ & $47.7 \pm 24.1$ \\
E/A & $1.68 \pm 0.28$ & $2.88 \pm 2.76$ & $3.40 \pm 2.24^{*}$ \\
EDT $(\mathrm{ms})$ & $49.5 \pm 6.8$ & $38.4 \pm 10.3^{*}$ & $38.7 \pm 13.3^{*}$ \\
IVRT $(\mathrm{ms})$ & $28.1 \pm 4.0$ & $22.4 \pm 4.6^{*}$ & $19.7 \pm 6.2^{*}$ \\
IVRTn & $58.6 \pm 15.4$ & $51.4 \pm 10.2$ & $44.5 \pm 13.9^{*}$ \\
\hline
\end{tabular}

Data are mean \pm standard deviation. Sham: control sham-operated rats; AS: aortic stenosis; AS-SPR: aortic stenosis treated with spironolactone; HR: heart rate; MWFS: midwall fractional shortening; EFS: endocardial fractional shortening; PWSV: posterior wall shortening velocity; E/A: early-to-late diastolic mitral inflow ratio; EDT: E-wave deceleration time; IVRT: isovolumetric relaxation time; IVRTn: isovolumetric relaxation time normalized to heart rate. ANOVA and Tukey.

* $\mathrm{p}<0.05$ vs Sham.

\# $\mathrm{p}<0.05$ vs AS.

worse systolic function than AS, characterized by its reduced PWSV and diastolic function, characterized by a higher E/A ratio. The more highly impaired systolic function in AS-SPR than AS was reinforced by endocardial fractional shortening, which was higher in AS than Sham, showing better systolic function in AS. In AS-SPR, this parameter had values between those in Sham and AS groups and did not significantly differ from either group, further characterizing the impairment of systolic function with spironolactone. Anatomic data confirmed greater LV hypertrophy and also showed greater right ventricular hypertrophy in AS-SPR than AS. In rats with LV pressure overload, right ventricular hypertrophy is considered a strong marker of LV failure and has been characterized by a right ventricle weight-to-body weight ratio greater than $0.8 \mathrm{mg} / \mathrm{kg}[34,35]$. Therefore, our data suggest that the AS-SPR group presented a more advanced degree of heart failure than AS.

As aldosterone is a potent fibrosis inductor, we quantified myocardial fibrosis by histologic and biochemical analysis. The AS had a higher interstitial collagen fractional area and hydroxyproline concentration than Sham, and in AS-SPR these parameters had values between those of the Sham and AS groups, being statistically similar to each group. These data suggest that spironolactone may have attenuated the development of myocardial fibrosis.

Matrix metalloproteinases are proteolytic enzymes responsible for extracellular matrix component degradation. MMP activation can occur in response to many stimuli including neurohormonal stimulation and myocardial stretching and precedes ventricular dilation in different

Table 4

Isolated papillary muscle basal data.

\begin{tabular}{llll}
\hline & $\begin{array}{l}\text { Sham } \\
(\mathrm{n}=13)\end{array}$ & $\begin{array}{l}\text { AS } \\
(\mathrm{n}=18)\end{array}$ & $\begin{array}{l}\text { AS-SPR } \\
(\mathrm{n}=12)\end{array}$ \\
\hline $\mathrm{DT}\left(\mathrm{g} / \mathrm{mm}^{2}\right)$ & $5.72 \pm 1.11$ & $4.51 \pm 1.11^{*}$ & $4.83 \pm 1.07^{*}$ \\
$\mathrm{RT}\left(\mathrm{g} / \mathrm{mm}^{2}\right)$ & $0.60 \pm 0.15$ & $0.79 \pm 0.21^{*}$ & $0.77 \pm 0.26^{*}$ \\
$\mathrm{RT} / \mathrm{TT}(\%)$ & $9.59 \pm 2.06$ & $15.3 \pm 4.33^{*}$ & $13.8 \pm 4.73^{*}$ \\
$\mathrm{TPT}(\mathrm{ms})$ & $160(159-160)$ & $165(160-170)$ & $180(168-200)^{*}$ \\
$+\mathrm{dT} / \mathrm{dt}\left(\mathrm{g} / \mathrm{mm}^{2} / \mathrm{s}\right)$ & $65.1 \pm 13.6$ & $46.0 \pm 12.8^{*}$ & $46.3 \pm 12.7^{*}$ \\
$-\mathrm{dT} / \mathrm{dt}\left(\mathrm{g} / \mathrm{mm}^{2} / \mathrm{s}\right)$ & $27.2 \pm 5.63$ & $23.4 \pm 6.02$ & $26.0 \pm 6.68$ \\
PM weight $(\mathrm{mg})$ & $8.02 \pm 1.84$ & $10.04 \pm 1.56$ & $9.43 \pm 3.11$ \\
$\mathrm{~L}_{\text {max }}(\mathrm{mm})$ & $7.60 \pm 1.05$ & $8.18 \pm 0.98$ & $8.13 \pm 1.06$ \\
PM CSA $\left(\mathrm{mm}^{2}\right)$ & $1.05 \pm 0.15$ & $1.23 \pm 0.19$ & $1.16 \pm 0.28$ \\
\hline
\end{tabular}

Data are mean \pm standard deviation or median and 25th and 75th percentiles. Sham: control sham-operated rats; AS: aortic stenosis; AS-SPR: aortic stenosis treated with spironolactone; DT: peak of developed tension; RT: resting tension; TT: total tension (RT + DT); TPT: time to peak of tension; + dT/dt: maximum rate of tension development; -dT/dt: maximum rate of tension decline; PM: papillary muscle; $\mathrm{L}_{\mathrm{max}}$ : muscle length at peak of the tension-length curve; CSA: cross-sectional area. ANOVA and Tukey or Kruskal-Wallis and Dunn.

$\mathrm{p}<0.05$ vs Sham. 
Table 5

Isolated papillary muscle data after positive inotropic stimulation.

\begin{tabular}{|c|c|c|c|c|}
\hline & & $\begin{array}{l}\text { Sham } \\
(n=13)\end{array}$ & $\begin{array}{l}\text { AS } \\
(n=18)\end{array}$ & $\begin{array}{l}\text { AS-SPR } \\
(n=12)\end{array}$ \\
\hline \multirow[t]{3}{*}{ PP60 } & DT $\left(\mathrm{g} / \mathrm{mm}^{2}\right)$ & $6.92(5.96-7.62)$ & $5.86(4.59-7.17)$ & $5.17(4.62-7.44)$ \\
\hline & $+\mathrm{dT} / \mathrm{dt}\left(\mathrm{g} / \mathrm{mm}^{2} / \mathrm{s}\right)$ & 81.4 (69.5-94.3) & $60.1(50.4-75.5)^{*}$ & $45.5(42.6-73.8)^{*}$ \\
\hline & TPT (ms) & 165 (160-170) & $168(165-180)$ & $188(175-195)^{*}$,\# \\
\hline \multirow{3}{*}{$2.5 \mathrm{mM}\left[\mathrm{Ca}^{2+}\right]_{0}$} & DT $\left(\mathrm{g} / \mathrm{mm}^{2}\right)$ & $6.38(5.57-7.18)$ & $5.19(4.31-6.51)$ & $5.10(4.53-6.83)$ \\
\hline & $+\mathrm{dT} / \mathrm{dt}\left(\mathrm{g} / \mathrm{mm}^{2} / \mathrm{s}\right)$ & $75.2 \pm 17$ & $60.0 \pm 16^{*}$ & $56.3 \pm 17^{*}$ \\
\hline & TPT (ms) & $150(144-160)$ & $160(150-160)$ & $175(163-183)^{*}$ \\
\hline \multirow[t]{3}{*}{$10^{-6} \mathrm{M}$ Iso } & DT $\left(\mathrm{g} / \mathrm{mm}^{2}\right)$ & $5.83 \pm 1.43$ & $4.21 \pm 1.27^{*}$ & $3.96 \pm 1.19^{*}$ \\
\hline & $+\mathrm{dT} / \mathrm{dt}\left(\mathrm{g} / \mathrm{mm}^{2} / \mathrm{s}\right)$ & $83.2 \pm 22.7$ & $54.1 \pm 18.9^{*}$ & $48.2 \pm 17.2^{*}$ \\
\hline & $\mathrm{TPT}(\mathrm{ms})$ & $120(120-121)$ & $130(120-140)$ & $140(120-140)^{*}$ \\
\hline
\end{tabular}

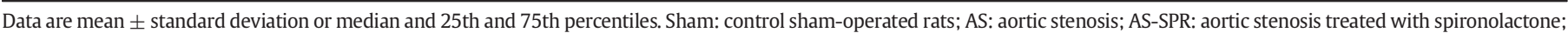

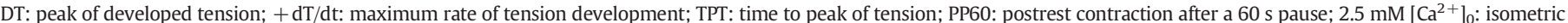

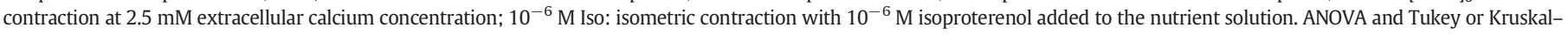
Wallis and Dunn.

* $\mathrm{p}<0.05$ vs Sham

\# $\mathrm{p}<0.05$ vs AS.

cardiac injury models $[45,46]$. In ventricular myocyte culture, aldosterone stimulates MMP activity [47], and in patients with ischemic heart failure, spironolactone reduces MMP-9 serum activity [48]. However, in this study we did not observe differences in MMP- 2 or -9 activation between groups. As we only evaluated MMP activation at the end of the experiment, we cannot exclude the influence of increased MMP activity during cardiac remodeling.

Most experimental studies have shown that aldosterone blockade has beneficial effects in different cardiac injury models. In pressure overloaded-induced cardiac remodeling, beneficial effects on different aspects of remodeling were observed when aldosterone blocker was initiated after LV hypertrophy had been established $[9,10,49]$. As aldosterone induces myocyte growth [2], we expected that aldosterone blockade would reduce cardiac hypertrophy. In fact, Pitt et al. [50] showed that aldosterone blocker eplerenone decreased LV hypertrophy in patients with systemic arterial hypertension. On the other hand, in patients with moderate-to-severe aortic stenosis, eplerenone did not change ventricular mass or the period without ventricular dysfunction [51].

Systolic wall stress can be calculated as intraventricular systolic pressure $\times$ cavity radius / 2 (LV wall thickness) [52]. When LV pressure is chronically increased, ventricular hypertrophy is initially important for reducing afterload and maintaining systolic function in the face of elevated intraventricular systolic pressure [52]. Cardiac hypertrophy is often accompanied by interstitial myocardial fibrosis. However, the role of hypertrophy and myocardial fibrosis during chronic pressure overload remains controversial $[15,16]$. These processes have been considered to have both beneficial and deleterious effects, according to stimulation characteristics such as overload intensity and duration, and onset severity [15].

In this study, spironolactone caused more maladaptive hypertrophy with more dilation, increased filling pressures, and more pronounced right ventricular hypertrophy, suggesting that spironolactone may be deleterious during early chronic pressure overload. The essential involvement of aldosterone in ventricular grow and development at an early age has not been established. We have not identified any studies evaluating the effects of early aldosterone blockade during chronic pressure overload. However, in different experimental models of transgenic or induced myocardial fibrosis inhibition, aortic banding was followed by reduced myocardial fibrosis and increased end-diastolic pressure or LV dilation [53-55]. Thus, our data and these results from literature allow us to hypothesize that, after elevation in LV pressure, the increase in myocardial interstitial collagen tissue is important in preventing ventricular chamber dilation. By inhibiting interstitial fibrosis, spironolactone may have facilitated ventricular dilation to occur early during chronic pressure overload. Ventricular dilation, per se, may be responsible for the impaired LV systolic and diastolic functions observed in the AS-SPR group. The papillary muscle preparations allow us to analyze myocardial function and contractility independent of the influence of afterload and preload. Data from papillary muscle functional study reinforces this hypothesis as, except for the increased time to peak of tension, myocardial contractility at basal conditions and after positive inotropic stimulation did not differ between AS-SPR and AS groups. Currently, as several drugs to prevent or reverse myocardial fibrosis are under investigation, it is important to better understand the role of connective tissue and fibrosis during the early phase of chronic pressure overload.

A limitation of this study refers to the fact that cardiac remodeling was only evaluated at the end of the experiment. Additional studies with different evaluation periods and detailed molecular analysis will be helpful in clarifying the physiopathological mechanisms involved in the effects of aldosterone blockade during early pressure overload. Furthermore, in this study, aortic stenosis was induced in young rats. As the hypertrophic response to a pressure overload present at birth may differ from the response to one acquired later in life [56], additional research is needed to establish whether our results are applicable to later acquired

Table 6

Left ventricular morphometric and biochemical parameters.

\begin{tabular}{|c|c|c|c|}
\hline & Sham & AS & AS-SPR \\
\hline Myocyte diameter ( $\mu \mathrm{m} ; \mathrm{n}=10 /$ group) & $12.75 \pm 2.06$ & $14.64 \pm 2.18^{*}$ & $16.35 \pm 2.68^{*}$ \\
\hline CFA (\%; $n=10 /$ group) & $2.29 \pm 0.77$ & $5.13 \pm 3.37^{*}$ & $3.14 \pm 3.25$ \\
\hline HOP (mg/g; $\mathrm{n}=10$ /group) & $2.62 \pm 0.57$ & $5.27 \pm 1.58^{*}$ & $3.29 \pm 0.26$ \\
\hline MMP-2 (59/pro MMP-2) & $2.76(1.58-3.03)$ & $1.69(1.61-2.14)$ & $1.75(1.59-1.87)$ \\
\hline MMP-2 (64/pro MMP-2) & $3.61(2.20-5.20)$ & $3.80(3.54-4.05)$ & $3.31(3.20-4.27)$ \\
\hline MMP-2 ([59 + 64]/pro MMP-2) & $5.78(4.81-7.84)$ & $5.65(5.26-5.91)$ & $5.16(4.69-5.93)$ \\
\hline MMP-9 (ativa/pro MMP-9) & $0.98(0.59-1.82)$ & $1.41(0.98-2.26)$ & $1.14(0.76-1.41)$ \\
\hline
\end{tabular}

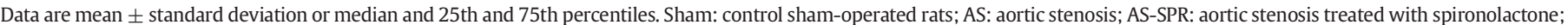

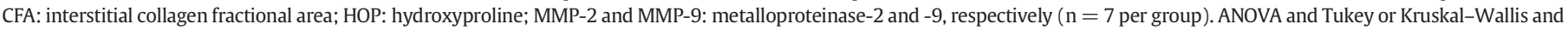
Dunn.

* $\mathrm{p}<0.05$ vs Sham. 

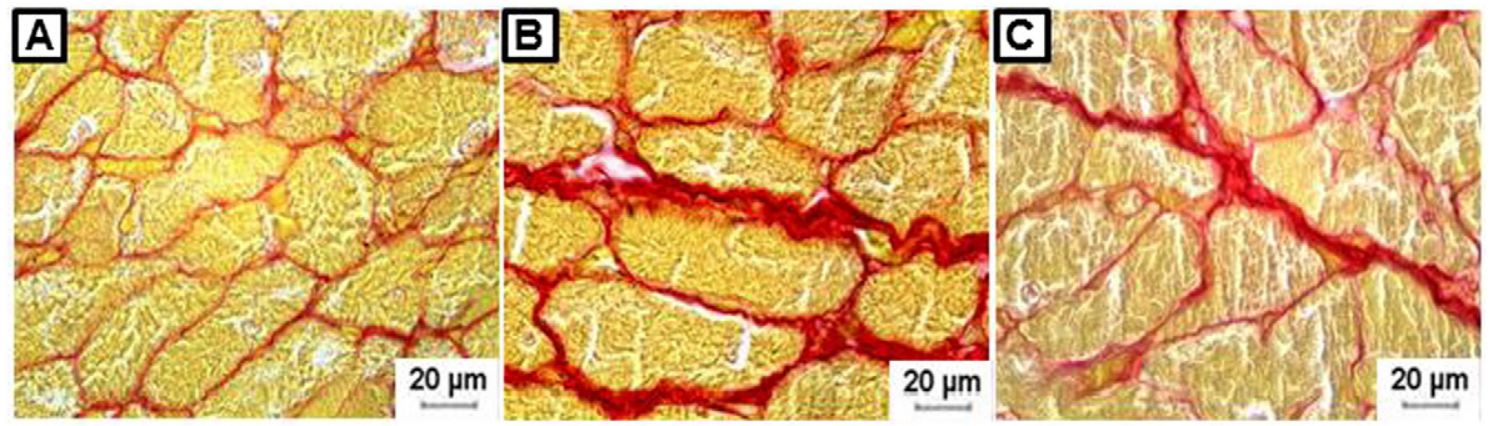

Fig. 2. Sirius red-stained myocardial histological sections. A: control sham-operated rats; B: aortic stenosis (AS); C: aortic stenosis treated with spironolactone (AS-SPR).

aortic stenosis. Nonetheless, caution is warranted when prescribing aldosterone blockers to patients in an early stage of aortic stenosis.

In conclusion, early administration of aldosterone antagonist spironolactone causes further hypertrophy in cardiac chambers, and left ventricular dilation and dysfunction without changing myocardial function in rats with aortic stenosis-induced chronic pressure overload.

\section{Conflict of interest}

The authors report no relationship that could be construed as a conflict of interest.

\section{Ethical statement}

We adhere to the statement of ethical publishing as appears in the International Journal of Cardiology [57].

\section{Grants}

Financial support was provided by CNPq (308674/2015-4, 306770/ 2015-6, 479085/2013-7, 480829/2013-6), FAPESP (2009/54506-7, 2015/17539-5), CAPES, and PROPe, UNESP.

\section{Acknowledgments}

The authors are grateful to Jose Carlos Georgette for their technical assistance and Colin Edward Knaggs for English editing.

\section{References}

[1] R.J. Mentz, G.L. Bakris, B. Waeber, J.J. McMurray, M. Gheorghiade, L.M. Ruilope, et al., The past, present and future of renin-angiotensin aldosterone system inhibition, Int. J. Cardiol. 167 (2013) 1677-1687.

[2] M.P. Okoshi, X. Yan, K. Okoshi, M. Nakayama, A.J.T. Schuldt, T. O'Connell, et al., Aldosterone directly stimulates cardiac myocyte hypertrophy, J. Card. Fail. 10 (2004) 511-518.

[3] S. Messaoudi, F. Azibani, C. Delcayre, F. Jaisser, Aldosterone, mineralocorticoid receptor, and heart failure, Mol. Cell. Endocrinol. 350 (2012) 266-272.

[4] M.B. Nolly, C.I. Caldiz, A.M. Yeves, M.C. Villa-Abrille, P.E. Morgan, N. Amado Mondaca, et al., The signaling pathway for aldosterone-induced mitochondrial production of superoxide anion in the myocardium, J. Mol. Cell. Cardiol. 67 (2014) $60-68$.

[5] B. Pitt, F. Zannad, W.J. Remme, R. Cody, A. Castaigne, A. Perez, et al., The effect of spironolactone on morbidity and mortality in patients with severe heart failure. Randomized Aldactone Evaluation Study (RALES) Investigators. N Engl J Med 341 (1999) 709-717.

[6] B. Pitt, W. Remme, F. Zannad, J. Neaton, F. Martinez, B. Roniker, et al., Eplerenone, a selective aldosterone blocker, in patients with left ventricular dysfunction after myocardial infarction, N. Engl. J. Med. 348 (2003) 1309-1321.

[7] F. Zannad, J.V. McMurray, H. Krum, D.J. van Veldhuisen, K. Swedberg, H. Shi, et al., Eplerenone in patients with systolic heart failure and mild symptoms, EMPHASIS -HF Study Group. N Engl J Med 364 (2011) 11-21.

[8] M.D. Cezar, R.L. Damatto, L.U. Pagan, A.R. Lima, P.F. Martinez, C. Bonomo, et al., Early spironolactone treatment attenuates heart failure development by improving myocardial function and reducing fibrosis in spontaneously hypertensive rats, Cell. Physiol. Biochem. 36 (2015) 1453-1466.
[9] N. Kobayashi, K. Yoshida, S. Nakano, T. Ohno, T. Honda, Y. Tsubokou, et al. Cardioprotective mechanisms of eplerenone on cardiac performance and remodeling in failing rat hearts, Hypertension 47 (2006) 671-679.

[10] G.M. Kuster, E. Kotlyar, M.K. Rude, D.A. Siwik, R. Liao, W.S. Colucci, et al., Mineralocorticoid receptor inhibition ameliorates the transition to myocardial failure and decreases oxidative stress and inflammation in mice with chronic pressure overload Circulation 111 (2005) 420-427.

[11] B. Martin-Fernandez, N. de Las Heras, M. Miana, S. Ballesteros, M. Valero-Munoz, D Vassallo, et al., Spironolactone prevents alterations associated with cardiac hypertrophy produced by isoproterenol in rats: involvement of SGK-1, Exp. Physiol. 97 (2012) 710-718.

[12] C.W. Yancy, M. Jessup, B. Bozkurt, J. Butler, D.E. Casey Jr., M.H. Drazner, et al., 2013 ACCF/AHA guideline for the management of heart failure: executive summary. A report of the American College of Cardiology Foundation/American Heart Association task force on practice guidelines, Circulation 128 (2013) 1810-1852.

[13] B. Pitt, M.A. Pfeffer, S.F. Assmann, R. Boineau, I.S. Anand, B. Claggett, et al., Spironolactone for heart failure with preserved ejection fraction, N. Engl. J. Med. 370 (2014) 1383-1392.

[14] F. Edelmann, R. Wachter, A.G. Schmidt, E. Kraigher-Krainer, C. Colantonio, W. Kamke, et al., Effect of spironolactone on diastolic function and exercise capacity in patients with heart failure with preserved ejection fraction: the ALDO-DHF randomized controlled trial, JAMA 309 (2013) 781-791.

[15] B. Crozatier, R. Ventura-Clapier, Inhibition of hypertrophy, per se, may not be a good therapeutic strategy in ventricular pressure overload: other approaches could be more beneficial, Circulation 131 (2015) 1448-1457.

[16] G.G. Schiattarella, J.A. Hill, Inhibition of hypertrophy is a good therapeutic strategy in ventricular pressure overload, Circulation 131 (2015) 1435-1447.

[17] A.C. Cicogna, K.G. Robinson, C.H. Conrad, K. Singh, R. Squire, M.P. Okoshi, et al., Direct effects of colchicine on myocardial function. Studies in hypertrophied and failing spontaneously hypertensive rats, Hypertension 33 (1999) 60-65.

[18] L.U. Pagan, R.L. Damatto, M.D. Cezar, A.R. Lima, C. Bonomo, D.H. Campos, et al., Longterm low intensity physical exercise attenuates heart failure development in aging spontaneously hypertensive rats, Cell. Physiol. Biochem. 36 (2015) 61-74.

[19] K. Okoshi, H.B. Ribeiro, M.P. Okoshi, B.B. Matsubara, G. Gonçalves, R. Barros, et al., Improved systolic ventricular function with normal myocardial mechanics in compensated cardiac hypertrophy, Jpn. Heart J. 45 (2004) 647-656.

[20] M.J. Gomes, P.F. Martinez, D.H.S. Campos, L.U. Pagan, C. Bonomo, A.R. Lima, et al., Beneficial effects of physical exercise on functional capacity and skeletal muscle oxidative stress in rats with aortic stenosis-induced heart failure, Oxidative Med. Cell. Longev. 2016 (2016) 8695716.

[21] V.O. Moreira, C.A. Pereira, M.O. Silva, S.L. Felisbino, A.C. Cicogna, K. Okoshi, et al., Growth hormone attenuates myocardial fibrosis in rats with chronic pressure overload-induced left ventricular hypertrophy, Clin. Exp. Pharmacol. Physiol. 36 (2009) 325-330.

[22] R.W. Souza, W.P. Piedade, L.C. Soares, P.A. Souza, A.F. Aguiar, I.J. Vechetti-Junior et al., Aerobic exercise training prevents heart failure-induced skeletal muscle atrophy by anti-catabolic, but not anabolic actions, PLoS One 9 (2014), e110020.

[23] A.R. Lima, P.F. Martinez, K. Okoshi, D.M. Guizoni, L.A. Zornoff, D.H. Campos, et al., Myostatin and follistatin expression in skeletal muscles of rats with chronic heart failure, Int J Exp Path 91 (2010) 54-62.

[24] J.E. Toblli, G. Cao, C. Rivas, J.F. Giani, F.P. Dominici, Intravenous iron sucrose reverses anemia-induced cardiac remodeling, prevents myocardial fibrosis, and improves cardiac function by attenuating oxidative/nitrosative stress and inflammation, Int J. Cardiol. 212 (2016) 84-91.

[25] K. Okoshi, J.R. Fioretto, M.P. Okoshi, A.C. Cicogna, F.F. Aragon, L.S. Matsubara, et al., Food restriction induces in vivo ventricular dysfunction in spontaneously hypertensive rats without impairment of in vitro myocardial contractility, Braz. J. Med. Biol Res. 37 (2004) 607-613.

[26] R.M. Lang, L.P. Badano, V. Mor-Avi, J. Afilalo, A. Armstrong, L. Ernande, et al., Recommendations for cardiac chamber quantification by echocardiography in adults: an update from the American Society of Echocardiography and the European Association of Cardiovascular Imaging, Eur Heart J Cardiovasc Imaging 16 (2015) 233-270.

[27] W.W. Parmley, D.L. Brutsaert, E.H. Sonnenblick, Effects of altered loading on contractile events in isolated cat papillary muscle, Circ. Res. 24 (1969) 521-532.

[28] C.M. Rosa, N.P. Xavier, D.H. Campos, A.A. Fernandes, M.D. Cezar, P.F. Martinez, et al., Diabetes mellitus activated fetal gene program and intensifies cardiac remodeling 
and oxidative stress in aged spontaneously hypertensive rats, Cardiovasc. Diabetol. 12 (2013) 152

[29] A.L. Gut, M.P. Okoshi, C.R. Padovani, F.F. Aragon, A.C. Cicogna, Myocardial dysfunction induced by food restriction is related to calcium cycling and beta-adrenergic system changes, Nutr. Res. 23 (2003) 911-919.

[30] M.P. Okoshi, L.S. Matsubara, M. Franco, A.C. Cicogna, B.B. Matsubara, Myocyte necrosis is the basis for fibrosis in renovascular hypertensive rats, Braz. J. Med. Biol. Res. 30 (1997) 1135-1144.

[31] J.R. Fioretto, S.S. Queiroz, C.R. Padovani, L.S. Matsubara, K. Okoshi, B.B. Matsubara, Ventricular remodeling and diastolic myocardial dysfunction in rats submitted to protein-calorie malnutrition, Am. J. Phys. 282 (2002) H1327-H1333.

[32] L.S. Matsubara, B.B. Matsubara, M.P. Okoshi, M. Franco, A.C. Cicogna, Myocardial fibrosis rather than hypertrophy induces diastolic dysfunction in renovascular hypertensive rats, Can. J. Physiol. Pharmacol. 75 (1997) 1328-1334.

[33] B.F. Polegato, M.F. Minicucci, P.S. Azevedo, R.F. Carvalho, F. Chiuso-Minicucci, E. J. Pereira, et al., Acute doxorubicin-induced cardiotoxicity is associated with matrix metalloproteinase-2 activation in rats, Cell. Physiol. Biochem. 35 (2015) 1924-1933.

[34] P.F. Martinez, K. Okoshi, L.A. Zornoff, S.A.J. Oliveira, D.H. Campos, A.R. Lima, et al. Echocardiographic detection of congestive heart failure in postinfarction rats, J. Appl. Physiol. 111 (2011) 543-551.

[35] O.H.L. Bing, W.W. Brooks, K.G. Robinson, M.T. Slawsky, J.A. Hayes, S.E. Litwin, et al., The spontaneously hypertensive rat as a model of the transition from compensated left ventricular hypertrophy to failure, J Mol Cell Cardiol 27 (1995) 383-396.

[36] P. Mulder, V. Mellin, J. Favre, M. Vercauteren, I. Remy-Jouet, C. Monteil, et al., Aldosterone synthase inhibition improves cardiovascular function and structure in rat with heart failure: a comparison with spironolactone, Eur. Heart J. 29 (2008) 2171-2179.

[37] J. Bauersachs, D. Fraccarollo, G. Ertl, N. Gretz, M. Wehling, M. Christ, Striking increase of natriuresis by low-dose spironolactone in congestive heart failure only in combination with ACE inhibition. Mechanistic evidence to support RALES, Circulation 102 (2000) 2325-2328.

[38] C.G. Brilla, L.S. Matsubara, K.T. Weber, Anti-aldosterone treatment and the prevention of myocardial fibrosis in primary and secondary hyperaldosteronism, J. Mol. Cell. Cardiol. 25 (1993) 563-575.

[39] M.D. Cezar, R.L. Damatto, P.F. Martinez, A.R. Lima, D.H. Campos, C.M. Rosa, et al., Aldosterone blockade reduces mortality without changing cardiac remodeling in spontaneously hypertensive rats, Cell. Physiol. Biochem. 32 (2013) 1275-1287.

[40] R.F. Carvalho, A.C. Cicogna, G.E.R. Campos, J.M.F. Assis, C.R. Padovani, M.P. Okoshi, et al., Myosin heavy chain expression and atrophy in rat skeletal muscle during transition from cardiac hypertrophy to heart failure, Int J Exp Path 84 (2003) 201-206.

[41] M.P. Okoshi, F.G. Romeiro, S.A. Paiva, K. Okoshi, Heart failure-induced cachexia, Arq. Bras. Cardiol. 100 (2013) 476-482.

[42] C. Helies-Toussaint, C. Moinard, C. Rasmusen, I. Tabbi-Anneni, L. Cynober, A Grynberg, Aortic banding in rat as a model to investigate malnutrition associated with heart failure, Am J Physiol Regulatory Integrative Comp Physiol 288 (2005) 1325-1331.

[43] S.F. Nagueh, O.A. Smiseth, C.P. Appleton, B.F. Byrd 3rd, H. Dokainish, T. Edvardsen, F.A. Flachskampf, T.C. Gillebert, A.L. Klein, P. Lancellotti, P. Marino, J.K. Oh, B.A. Popescu, A.D. Waggoner, Recommendations for the evaluation of left ventricular diastolic function by echocardiography: an update from the American Society of
Echocardiography and the European Association of Cardiovascular Imaging, J. Am. Soc. Echocardiogr. 29 (2016) 277-314.

[44] S. Kobayashi, M. Yano, M. Kohno, M. Obayashi, Y. Hisamatsu, T. Ryoke, T. Ohkusa, K. Yamakawa, M. Matsuzaki, Influence of aortic impedance on the development of pressure-overload left ventricular hypertrophy in rats, Circulation 94 (1996) 3362-3368.

[45] N. Nishikawa, K. Yamamoto, Y. Sakata, T. Mano, J. Yoshida, T. Miwa, et al., Differential activation of matrix metalloproteinases in heart failure with and without ventricular dilatation, Cardiovasc. Res. 57 (2003) 766-774.

[46] F.G. Spinale, Myocardial matrix remodeling and the matrix metalloproteinases: influence on cardiac form and function, Physiol. Rev. 87 (2007) 1285-1342.

[47] M.K. Rude, T.A.S. Duhaney, G.M. Kuster, S. Judge, J. Heo, W.S. Colucci, et al., Aldosterone stimulates matrix metalloproteinases and reactive oxygen species in adult rat ventricular cardiomyocytes, Hypertension 46 (2005) 555-561.

[48] M.J. Li, C.X. Huang, E. Okello, T. Yanhong, S. Mohamed, Treatment with spironolactone for 24 weeks decreases the level of matrix metalloproteinases and improves cardiac function in patients with chronic heart failure of ischemic etiology, Can J Cardiol 25 (2009) 523-526.

[49] K. Nagata, K. Obata, J. Xu, S. Ichihara, A. Noda, H. Kimata, et al., Mineralocorticoid receptor antagonism attenuates cardiac hypertrophy and failure in low-aldosterone hypertensive rats, Hypertension 47 (2006) 656-664.

[50] B. Pitt, N. Reichek, R. Willenbrock, F. Zannada, R.A. Phillips, B. Roniker, et al., Effects of eplerenone, enalapril, and eplerenone/enalapril in patients with essential hypertension and left ventricular hypertrophy. The 4E-Left Ventricular Hypertrophy Study, Circulation 108 (2003) 1831-1838.

[51] R.A.H. Stewart, A.J. Kerr, B.R. Cowan, A.A. Young, C. Occleshaw, A.M. Richards, et al., A randomized trial of the aldosterone-receptor antagonist eplerenone in asymptomatic moderate-severe aortic stenosis, Am. Heart J. 156 (2008) 348-355.

[52] L.H. Opie, G. Hasenfuss, Mechanisms of cardiac contraction and relaxation, Braunwald's Heart Disease. A Textbook of Cardiovascular Medicine, Elsevier Saunders, Philadelphia 2012, pp. 459-486

[53] M.A. Allwood, R.T. Kinobe, L. Ballantyne, N. Romanova, L.G. Melo, C.A. Ward, et al., Heme oxygenase- 1 overexpression exacerbates heart failure with aging and pressure overload but is protective against isoproterenol-induced cardiomyopathy in mice, Cardiovasc. Pathol. 23 (2014) 231-237.

[54] K.V. Engebretsen, K. Skardal, S. Bjornstad, H.S. Marstein, B. Skrbic, I. Sjaastad, et al., Attenuated development of cardiac fibrosis in left ventricular pressure overload by SM16, an orally active inhibitor of ALK5, J. Mol. Cell. Cardiol. 76 (2014) 148-157.

[55] J.A. Lucas, Y. Zhang, P. Li, K. Gong, A.P. Miller, E. Hassan, et al., Inhibition of transforming growth factor-beta signaling induces left ventricular dilation and dysfunction in the pressure-overloaded heart, Am. J. Physiol. Heart Circ. Physiol. 298 (2010) H424-H432.

[56] M.E. Assey, T. Wisenbaugh, J.F. Spann Jr., P.C. Gillette, B.A. Carabello, Unexpected persistence into adulthood of low wall stress in patients with congenital aortic stenosis: is there a fundamental difference in the hypertrophic response to a pressure overload present from birth? Circulation 75 (1987) 973-979.

[57] L.G. Shewan, G.M.C. Rosano, M.Y. Henein, A.J.S. Coats, A statement on ethical standards in publishing scientific articles in the International Journal of Cardiology family of journals, Int. J. Cardiol. 170 (2014) 253-254. 